\title{
Pediatría
}

http://www.revistapediatria.org/

DOI: https://10.14295/rp.v54i2.152

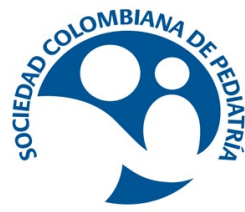

Regional Bogotá

Reportes de caso

\section{Onfalitis y Quiste de Cordón Umbilical asociada a persistencia del uraco en un recién nacido.}

\section{Ricardo Hernández-Sarmiento ${ }^{a}$, Álvaro Marroquín Murcia ${ }^{a}$, Christian Andrés Casas Cárdenas ${ }^{b}$, Adriana María Fajardo Cardonac.}

a. Residente de pediatría, Universidad del Rosario, Bogotá DC, Colombia

b. Pediatra, Hospital Mederi, Bogotá DC, Colombia.

c. Fellow de Neonatología, Universidad Militar Nueva Granada, Bogotá DC, Colombia

INFORMACIÓN DEL ARTÍCULO

Historia del artículo:

Recibido el 11 agosto 2020

Aceptado el 20 agosto 2021

Palabras clave:

Recién nacido

Uraco

Cordón umbilical

Ultrasonografía
R E S U M E N

Antecedentes: la persistencia del uraco es una patología poco frecuente. Su diagnóstico y tratamiento oportuno puede impactar en el pronóstico. Reporte de caso: recién nacido a término de 8 días, antecedente de quiste de cordón umbilical, que consulta por cuadro onfalitis, se inició manejo antimicrobiano, se realizó ecografía abdominal evidenciándose imagen compatible con persistencia del uraco. Fue llevada a cirugía con adecuada evolución clínica y completó cubrimiento antibiótico para Klebsiella pneumoniae y Staphylococcus aureus de manera favorable. Conclusión: La persistencia del uraco es una patología poco frecuente que requiere un manejo multidisciplinar para iniciar un tratamiento de manera oportuna. Los quistes de cordón umbilical y la onfalitis están asociados a la persistencia del uraco.

Omphalitis and umbilical cord cyst associated with patent urachus in a newborn

\begin{abstract}
A B S T R A C T
Background: Patent urachus is a rare pathology. Its diagnosis and timely treatment can have an impact on the prognosis. Case report: A term newborn of 8 days, history of Umbilical cord cyst, who consulted for omphalitis, for which antimicrobial treatment was started, abdominal ultrasound was performed, evidencing an image compatible with patent urachus. She was taken to surgery with adequate clinical evolution and completed antibiotic coverage for Klebsiella pneumoniae and Staphylococcus aureus favorably. Conclusion: Patent urachus is a rare pathology that requires multidisciplinary management to initiate treatment on time. The umbilical cord cysts and omphalitis are associated with patent urachus.
\end{abstract}

Keywords:

Newborn

Urachus

Umbilical cord

Ultrasonography

\footnotetext{
*Autor para correspondencia. Ricardo Hernández-Sarmiento Correo electrónico: rhernandezs15@hotmail.com
}

Como Citar: Onfalitis y Quiste de Cordón Umbilical asociada a persistencia del uraco en un recién nacido. Hernández-Sarmiento R, Marroquín Murcia A, Casas Cárdenas CA, Fajardo Cardona AM. Pediatr. 2021;54(2):78-81. 


\section{Introducción}

El uraco es una estructura que conecta la vejiga a la pared abdominal al nivel del ombligo, la cual. posterior al nacimiento se oblitera originando el ligamento umbilical interno (1). La prevalencia de las anomalías del uraco se aproxima al $1 \%$, siendo la principal la persistencia del uraco (PU) (2). La PU puede generar diferentes problemas al paciente tanto en la etapa neonatal como en niños mayores, como secreción umbilical, infección local, dolor abdominal e infección del tracto urinario $(3,4)$. Su diagnóstico requiere de imágenes como la ecografía y la tomografía y su tratamiento generalmente es quirúrgico, aunque se debe individualizar en cada paciente. $(3,5)$.

\section{Reporte de caso}

Paciente recién nacido a término, femenino, quien al nacimiento presentó quiste del cordón umbilical (Figura 1), por lo cual se realizó ecografía abdominal en donde no se evidenciaron anomalías. Consultó a los ocho días de vida por cuadro de onfalitis y conjuntivitis sin clínica de sepsis. Se inició cubrimiento antibiótico, sin embargo, por antecedente de quiste en cordón umbilical e infección local, se realizó nuevamente una ecografía abdominal en la cual se observó una estructura de morfología tubular, hipoecoica en línea media abdominal que comunica el domo de la vejiga con la región umbilical de aproximadamente $20 \mathrm{~mm}$ de trayecto, sin salida de contenido líquido a través del ombligo (Figura 2); lo cual sugiere como diagnóstico la PU. La paciente fue llevada a cirugía, confirmándose el diagnóstico, donde se encontró remanente del uraco que finaliza en un divertículo a nivel del domo vesical de $4 \mathrm{~mm}$ de diámetro, se realizó resección de dicho remanente, cistorrafia y onfaloplastia. Se completó antibioticoterapia endovenosa por aislamiento de Klebsiella pneumoniae y Staphylococcus aureus multisensibles en el cultivo de la secreción umbilical, se mantuvo con sonda vesical por tres días posquirúrgicos y egresó en buen estado general.

\section{Discusión}

El uraco es una estructura que solo está presente en la etapa embrionaria, la cual conecta la vejiga fetal y el alantoides localizado en el espacio preperitoneal en un compartimiento formado por la fascia umbilico-vesical y está acompañado por las arterias umbilicales a cada lado $(3,6)$. Al descender la vejiga entre el cuarto y el quinto mes de gestación a través de la pelvis, se estrecha dicho tejido, llevando así a la posterior obliteración de su lumen, generando como resultado el ligamento mediano umbilical en el domo de la vejiga $(7,8)$. En algunas ocasiones este proceso de obliteración es incompleto, creando una PU. La prevalencia de las anomalías del uraco se aproxima al $1 \%$, siendo la principal la PU (2) en un $50 \%$, de los casos, seguido de quiste de uraco en el $30 \%$ de los pacientes, del divertículo en el 3 al $5 \%$ de los casos y por el seno uracoumbilical en el $15 \%$. Esta malformación representa 1 de cada
200000 nacidos vivos, siendo dos veces más común en hombres que en mujeres (3).

El remanente del uraco se divide en cinco grupos, 1) La PU, que es la comunicación entre ombligo y vejiga; 2) El seno uracal que se desarrolla cuando el final del ombligo está abierto pero no existe comunicación con la vejiga; 3) El divertículo de uraco que es cuando forma un tapón en el domo de la vejiga; 4) El quiste del uraco cuando la parte central del tracto es un patente y está lleno de fluido y 5) El remanente atrésico del uraco que es cuando el tracto entero persiste como un cordón (7).

La PU suele sospecharse en ombligos que presenten drenaje y apariencia anormal en el periodo neonatal, también se puede evidenciar edema umbilical, granuloma y curación retardada del cordón umbilical. Por otro lado, el quiste de uraco suele presentarse con infección o dolor abdominal en la niñez tardía o adultez (6) pero la gran mayoría suelen ser asintomáticos (3).

En una revisión de 45 niños con promedio de edad de 4 años sin diferencia de sexo, que presentaban anormalidades a nivel del uraco, se encontró que el síntoma más común fue la secreción umbilical (42\%), seguido por la presencia de masa o quiste (33\%) y dolor (22\%), mientras que los síntomas urinarios fueron infrecuentes (<5\%) (9). La complicación más frecuente de la PU es la infección, la cual ocurre en el $23 \%$ de los casos, de tal forma que la mayoría de las PU suelen diagnosticarse durante un evento infeccioso, como en el caso reportado, aunque también puede confundirse con patologías inflamatorias abdominales y pélvicas o con tumores malignos. Estos procesos infecciosos pueden transmitirse por vía linfática, hematógeno o vesical, donde se pueden cultivar gérmenes Gram positivos y Gram negativos, siendo el Staphylococcus aureus el germen más comúnmente aislado, en un $82 \%$ de los casos (3), mientras que otros gérmenes también frecuentemente aislados son Escherichia coli, Enterococcus, Citrobacter, Klebsiella y Proteus (6).

El diagnóstico suele ser un hallazgo incidental, en un estudio realizado en Japón en 3400 niños menores a 15 años, 56 fueron diagnosticados de manera incidental con PU (1.6\%) por medio de ecografía abdominal (5). Esta indicado realizar ultrasonografía en todo paciente con sospecha de alteración en el uraco (9), ya que es la técnica más apropiada como aproximación dado su localización en la parte anterior del abdomen, lejos de la interfaz del gas intestinal (8). En la PU se suele ver una estructura tubular entre vejiga y ombligo, el seno uracoumbilical como una estructura tubular delgada sobre la línea media por debajo del ombligo y el quiste del uraco se aprecia como una lesión extra luminal que protruye de la vejiga, llena de líquido y no relacionada con el ombligo (3).

En el presente caso, se realizó ultrasonido dada la presencia del quiste del cordón umbilical, sin embargo, no fue inicialmente identificado, esto puede deberse a la naturaleza operador dependiente del ultrasonido. La tomografía presenta ciertas ventajas sobre la ultrasonografía, ya que posee mayor exactitud diagnóstica al permitir visualizar y delimitar la cavidad quística uracal, así como de otras estructuras adyacentes, logrando alcanzar diagnósticos diferenciales con otras patologías (8). También se deben contemplar otras pruebas diagnósticas en búsqueda de alteraciones asociadas o concomitantes, como el reflujo vesicoureteral, riñón único, criptorquidia, síndrome de la unión pieloureteral o hidronefrosis, las cuales se pueden 
Figura 1. Quiste de cordón umbilical.

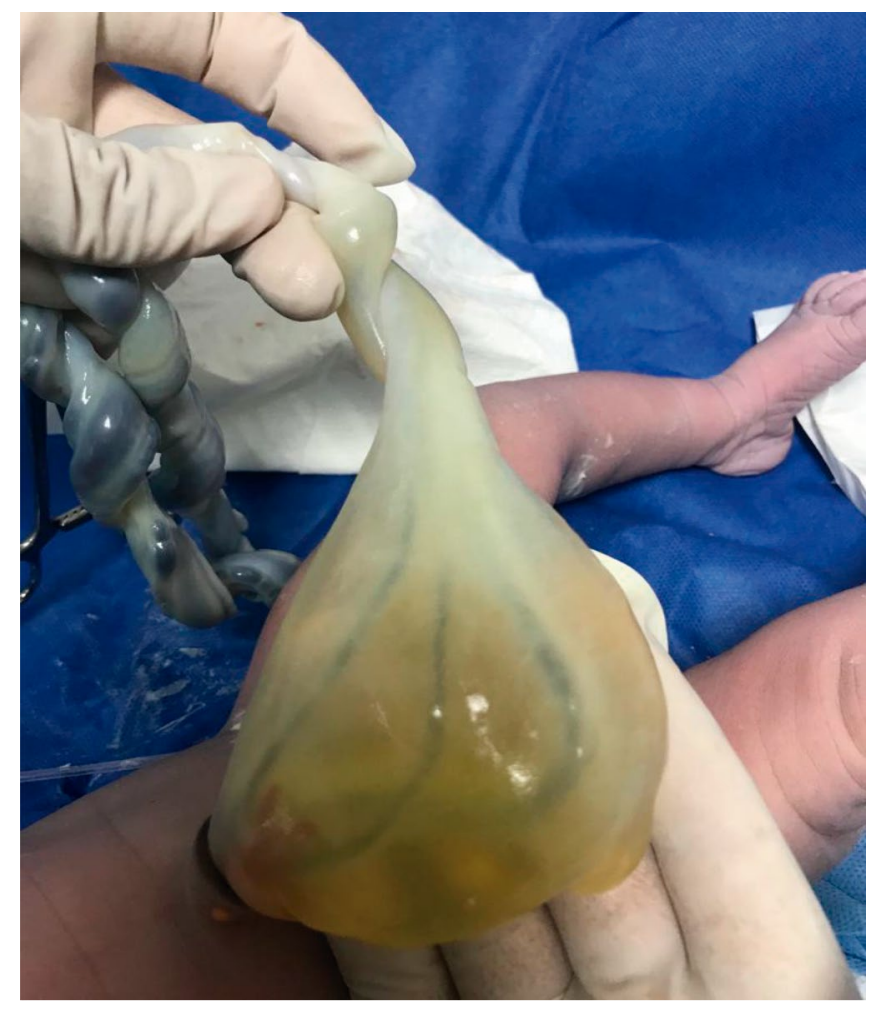

Figura 2. Ultrasonografía que muestra la persistencia del uraco.

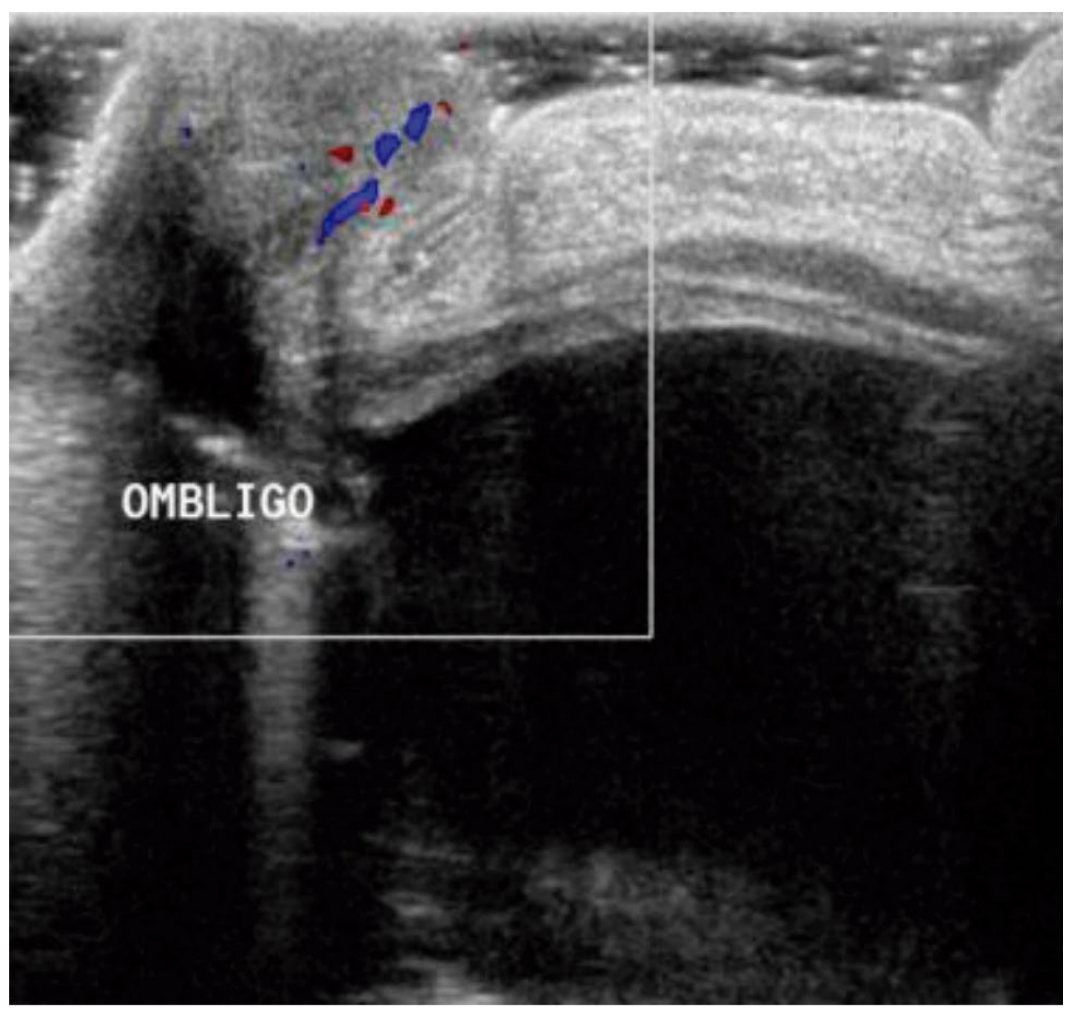

encontrar en un $43 \%$ de los pacientes como anomalías de uraco (8). Entre estos estudios están la urografía intravenosa, la cistouretrografía miccional para analizar la zona de comunicación entre la vejiga y el uraco y si existe una posible compre- sión a nivel de la cúpula de la vejiga, esta última presentándose hasta en el $14 \%$ de los pacientes $(6,8)$. Aunque la mayoría de los casos la PU se diagnostica en la vida postnatal, también 
puede ser diagnosticada en vida prenatal mediante una ultrasonografía o estudio de resonancia magnética fetal (6).

Los diagnósticos diferenciales incluyen anomalías del ducto vitelino, apendicitis, tejidos de granulación umbilicales $u$ onfalitis, este último suele presentarse como celulitis superficial y finalmente debe tenerse como otra posibilidad el remanente del conducto onfalomesentérico el cual se presenta como drenaje umbilical purulento $(3,6)$.

El tratamiento de elección es la escisión quirúrgica, aunque puede resolver de forma espontánea en menores de 6 meses. Sin embargo, en este grupo de edad se han reportado drenajes con catéter uretral a corto tiempo con cierre espontáneo de la PU $(6,7)$. En niños pequeños se suele realizar una incisión curvilínea infraumbilical y en niños más grandes una incisión transversa entre el ombligo y el pubis, posteriormente se remueve el tracto o quiste con una pequeña porción de la vejiga en el sitio de inserción. En manos de cirujanos expertos y en pacientes cuidadosamente seleccionados, la resección vía laparoscópica ha sido exitosa, proporcionando así una sanación más rápida y una menor estancia hospitalaria.(7,8).

Cuando se diagnostica la PU durante un episodio infeccioso, se recomienda realizar la corrección en dos tiempos quirúrgicos, puesto que se han reportado en algunas series, más de 40 \% de complicaciones de tipo infeccioso en las heridas quirúrgicas en los pacientes llevados a cirugía en un solo tiempo quirúrgico (3). Sin embargo, en el caso presentado, no se presentaron complicaciones a pesar de haberse realizado la corrección en un tiempo quirúrgico.

\section{Conclusiones}

La persistencia del uraco es una patología poco frecuente que puede afectar de manera importante la salud del paciente si no se diagnostica y trata a tiempo. Está asociado a los quistes de cordón umbilical y onfalitis tal como el caso presentado. Es indispensable un manejo interdisciplinar en conjunto con pediatría, radiología, urología y cirugía pediátrica, por lo que es importante conocer las manifestaciones clínicas para así poder iniciar una conducta de manera precoz.

\section{R E F E R E N C I A S}

1. Vargas M, Martínez L, Baquero F. Anomalía del uraco sobreinfectada como causa de irritabilidad en un lactante. Rev Pediatr Aten Primaria. 2016;18:259-62.

2. Vavilov S, Krishnan J, Jiwane A, Shand AW. Patent urachus with bladder prolapse. J Pediatr Surg Case Reports. 2017;24:17-20.

3. Orjuela C, Corredor H, Malo G. Patología del uraco Serie de casos. Rev Urol Colomb. 2010;19(2):65-71.

4. Widni EE, Höllwarth ME, Haxhija EQ. The impact of preoperative ultrasound on correct diagnosis of urachal remnants in children. J Pediatr Surg. 2010;45(7):1433-7.

5. Ueno T, Hashimoto H, Yokoyama H, Ito M, Kouda K, Kanamaru H. Urachal anomalies: Ultrasonography and management. J Pediatr Surg. 2003;38(8):1203-7.

6. Arlen AM, Smith EA. Disorders of the Bladder and Cloacal Anomaly. Clin Perinatol. 2014;41(3):695-707.

7. Naiditch JA, Radhakrishnan J, Chin AC. Current diagnosis and management of urachal remnants. J Pediatr Surg. 2013;48(10):2148-52.

8. Donate M, Giménez J, Salinas A, Lorenzo J, Segura M, Hernández I, et al. Patología del uraco: revisión de conjunto y presentación de tres casos. Actas Urol Esp. 2005;29(3):332-6.

9. Snyder CL. Current management of umbilical abnormalities and related anomalies. Semin Pediatr Surg. 2007;16:41-9. 